\section{P118 Cooperative Extension as a Model for Sustainable Dissemination of the Diabetes Prevention Program}

Traci L. Armstrong Florian, MS, RD, tarmstro@cals.arizona.edu, University of Arizona, 4341 East Broadway Road, Phoenix, AZ 85040; Hope Wilson, MPH, RDN; Melissa Wyatt, MS, RD; Joyce Alves, MS, RD; Martina Sepulveda, BS; Vanessa da Silva, PhD, RD

Objective: To demonstrate how Cooperative Extension (CE) can be used to successfully disseminate the National Diabetes Prevention Program (DPP).

Target Audience: Adults at risk for type 2 diabetes. Theory, Prior Research, Rationale: In the U.S., 30.3 million have diabetes, 84.1 million have prediabetes. The impact of diabetes is most strongly felt in medically underserved areas (MUAs), where healthcare access is limited. DPP has robust research supporting its success in reducing or delaying the onset of type 2 diabetes. However, the program's dissemination to MUAs is constrained by lack of trained personnel and access. The CE System addresses these constraints. CE has offices in most counties across the U.S., staffed with trained educators. Additionally, other Extension programming may complement and enhance the lifestyle outcomes encouraged in the DPP. The CE National Framework highlights chronic disease prevention and management as a priority. Thus, CE is an excellent fit for DPP to achieve a broader impact.

Description: DPP is a group, lifestyle-based intervention offered over a year's time, with 16 weekly/bi-weekly/ hour-long sessions in months 1-6, followed by 6-10 sessions during months 7-12. The evidence-based CDC curriculum, PreventT2, promotes healthy eating, physical activity, and modest weight loss.

Evaluation: A study, through pre/post surveys, showed those receiving counseling and motivational support on diet, exercise, and behavior modification by attending DPP-reduced their risk of diabetes by $58 \%$. Lifestyle changes for participants aged $60+$, reduced their risk by $71 \%$. Program success is evaluated by measuring primary outcomes determined by the CDC: change in body weight and increase in weekly minutes of physical activity.

Conclusions and Implications: Nationally, process evaluation results optimize DPP participant retention and success. The CDC DPP Impact Toolkit is used to show health and economic effects of CE-DPP. Combined, the strategies shared by this university's CE will be useful to other land-grant universities wishing to implement a CE-DPP.

Funding: University of Arizona, Cooperative Extension Funds.

\section{P119 Development and Validation of the ASKFV-STC Self-Efficacy Questionnaire for Requesting Fruits and Vegetables}

Kate Balestracci, MS, RDN, katebal12@uri.edu, University of Rhode Island, 80 Washington Street, Providence, RI 02903; Jade McNamara, MS; Geoffrey Greene, PhD, LD, RDN

Background (Background, Rationale, Prior Research, and/or Theory): Elementary-aged students' skills in asking for preferred fruit and vegetables (FV) in the school and home environment are associated with increased consumption of FV. Self-efficacy is associated with skill development, but there are limited instruments measuring this construct.

Objective: To develop an assessment tool, ASKFV-STC, measuring elementary-aged students' self-efficacy towards requesting $\mathrm{FV}$ in the school and home environment for the Students Take Charge! intervention study.

Study Design, Setting, Participants, Intervention: The self-efficacy tool development included items from existing self-efficacy surveys on asking and shopping for FV. Cognitive interviews (CI) were conducted at a predominately low-income (95.3\% free/reduced meals), ethnically diverse (83\% Hispanic, $8 \%$ black) school with 5 th grade males $(\mathrm{n}=10)$ and females $(\mathrm{n}=7)$. Survey field-testing data with low-income (88.3\% free/reduced meals), ethnically diverse (66.1\% Hispanic, 16.2\% black) children were used for exploratory (EFA) and confirmatory factor analysis (CFA). Outcome Measures and Analysis: Items were removed or modified based on CI feedback. Students were randomly split to conduct EFA $(n=176)$ and CFA $(n=186)$. Scales were assessed for reliability using Cronbach's alpha. Results: CI reduced the self-efficacy tool from 10 items to seven. The 7-item questionnaire was tested on a total of 444 fourth and fifth graders. EFA revealed that the items loaded on two factors: home self-efficacy (4 items) and school self-efficacy ( 2 items); one item was excluded based on low loading $(<.40)$. The reduced 6-item, 2-factor structure was the best fit for the data $(\chi=45.09$, df $=9$; $\mathrm{CFI}=0.835$; RMSEA $=0.147)$. The 4 -item home selfefficacy was found to have high reliability $(a=.73)$, along with a marginally acceptable reliability for the 2-item school self-efficacy $(\mathrm{a}=.53)$.

Conclusions and Implications: The ASKFV-STC selfefficacy questionnaire is a novel, brief instrument that measures children's self-efficacy for asking for FVs in the home and school environments. Strengths included: comprehensive instrument development process, strong psychometric properties, and low participant burden. Future steps include development of items to more reliably measure school self-efficacy.

Funding: Supplemental Nutrition Assistance Program-Education. 